\title{
ANALYZING THE DYNAMICS OF THE FORCED BURGERS EQUATION
}

\author{
NEJIB SMAOUI \\ Kuwait University \\ Department of Mathematics and Computer Science \\ P.O. Box 5969, Safat 13060, Kuwait
}

(Received April, 1998; Revised October, 1999)

\begin{abstract}
We study numerically the long-time dynamics of a system of reaction-diffusion equations that arise from the viscous forced Burgers equation $\left(u_{t}+\right.$ $\left.u u_{x}-\nu u_{x x}=F\right)$. A nonlinear transformation introduced by Kwak is used to embed the scalar Burgers equation into a system of reaction diffusion equations. The Kwak transformation is used to determine the existence of an inertial manifold for the 2-D Navier-Stokes equation. We show analytically as well as numerically that the two systems have a similar, long-time dynamical, behavior for large viscosity $\nu$.
\end{abstract}

Key words: Parabolic Equation, Reaction-Diffusion Equations, Inertial Manifold, Kwak Transformation.

AMS subject classifications: $35 \mathrm{~F} 25,76 \mathrm{R} 50$.

\section{Introduction}

In recent years, there has been growing interest in studying dynamical systems that arise from solving the initial value problems for nonlinear partial differential equations. Starting in the 1970's, similarities between the theories of ODEs and PDEs have been observed in the context of the qualitative theory of differential equations, especially in the case of parabolic PDEs. Henry [12] gives various examples of this trend, comparing the stability properties of PDEs to those of ODEs. Later, the work of J. Mallet-Paret [17], Mañé [18], and others opened up new avenues for understanding the long time dynamics of a more general class of dissipative PDEs.

A similarity between the two fields were further strengthened by the results of Babin, Vishik, Constantin, Foias, Temam, and Ladyzhenskaya $[1,7,10,15,20]$ that proved the finite dimensionality of the global attractor for the 2-D Navier-Stokes (N$\mathrm{S})$ equations. Because of the importance of the N-S equations in aerodynamics, oceandynamics, fluid mechanics, and hydrodynamic stability, the finite dimensionality of its attractor suggests that the dynamics on the attractor can be captured by a system of ODEs. Hence, the long-time dynamics of the PDEs is equivalent in some sense to the dynamics of a suitable system of ODEs. Smaoui [19] has shown that the dynam- 
ics of Kolmogorov flow is equivalent to the dynamics of a system of ODEs for a certain parameter range. The notion of inertial manifold was introduced by Foias, Sell and Temam [9] as a way to obtain such a system of ODEs. Subsequently, various attempts have been made to exhibit inertial manifolds for a large class of PDEs [8].

More strikingly yet, even in the case of the scalar viscous Burgers equation, due to the non-availability of the spectral gap condition, the existence of an inertial manifold remained an open problem. Recently, Kwak [14] introduced a nonlinear transformation that embeds the scalar Burgers equation into a system of reaction-diffusion equations that admit an inertial manifold. The Kwak transformation is briefly summarized in Section 2. Until now, the nature of this transformation has not been studied numerically. In particular, the dynamics of the scalar viscous Burgers equation have not been compared with those of the reaction-diffusion system that arises before adding additional corrective camping terms. The work reported herein describes a numerical study of the two PDE systems without the additional corrective terms.

The remainder of this paper is organized as follows: In Section 2, we briefly introduce the Kwak Transformation. Section 3 discusses some analytical results of both, the forced scalar Burgers equation and the transformed reaction-diffusion system. Section 4 shows the numerical results of both of these equations which supports the analytical ones.

\section{The Kwak Transformation}

The viscous Burgers equation

$$
u_{t}+u u_{x}-\nu u_{x x}=0
$$

with periodic boundary conditions $u(2 \pi, t)=u(0, t)$ and given initial value $u(x, 0)=$ $u_{0}(x)$ is a well known and well understood quasilinear parabolic equation. It first appeared in a paper by Bateman [2] and was used extensively by Burgers [4, 5] as a simple model for turbulent liquid flow through a channel. Burgers equation was also used to model certain gas dynamics [16] and acoustic waves [3]. A complete solution for Equation (1) is presented by Hopf [13]. In the present paper, we study the forced Burgers equation where the force is sinusoidal

$$
u_{t}+u u_{x}-\nu u_{x x}=F(x)
$$

Equation (2) can be transformed by the transformation $J(u)=\left(u, u_{x},-\frac{1}{2} u^{2}\right)$, with $v=u_{x}$, and $w=-\frac{1}{2} u^{2}$, into the system

$$
\begin{gathered}
u_{t}=\nu u_{x x}+w_{x}+F(x) \\
v_{t}=\nu v_{x x}+w_{x x}+F^{\prime}(x) \\
w_{t}=\nu w_{x x}+\nu v^{2}+u^{2} v-u F(x)
\end{gathered}
$$

with periodic boundary conditions $u(2 \pi, t)=u(0, t), v(2 \pi, t)=v(0, t)$, and $w(2 \pi, t)=$ $w(0, t)$. The given initial conditions are specified as $u(x, 0)=u_{0}(x), v(x, 0)=v_{0}(x)$, and $w(x, 0)=w_{0}(x)$. 
This transformation is utilized in a slightly different way than that used by Kwak [14]. Kwak, when studying the asymptotic dynamics of a class of quasilinear parabolic equations given by

$$
u_{t}=u_{x x}+(f(u))_{x}+g(u)+h(x)
$$

on the interval $[0, L]$, introduces a nonlinear change of variables to transform Equation (4) into a reaction-diffusion system. The transformation is defined by

$$
J(u)=\left(u, u_{x}, f(u)\right)
$$

so that $(u, v, w)=J(u)$ satisfies the system of equations

$$
\begin{gathered}
u_{t}=u_{x x}+w_{x}+g(u)+h(x) \\
v_{t}=v_{x x}+w_{x x}+g^{\prime}(u) v+h^{\prime}(x) \\
w_{t}=w_{x x}-f^{\prime \prime}(u) v^{2}+f^{\prime}(u)^{2} v+f^{\prime}(u)\{g(u)+h(x)\}
\end{gathered}
$$

with the periodic boundary condition given by $J(u(0, t))=J(u(2 \pi, t))$ and initial values given by $J\left(u_{0}(x)\right)$. In $(6)$, the prime denotes the derivative of the corresponding function. We apply this transformation to the forced Burgers equation

$$
u_{t}=u_{x x}-u u_{x}+h(x)
$$

where $h(x)=F(x) / \nu^{2}$, by setting $u=u, v=u_{x}$ and $w=-\frac{1}{2} u^{2}$ and obtain

$$
\begin{gathered}
u_{t}=u_{x x}+w_{x}+h(x) \\
v_{t}=v_{x x}+w_{x x}+h^{\prime}(x) \\
w_{t}=w_{x x}+v^{2}+u^{2} v-u h(x) .
\end{gathered}
$$

\section{Analytical Results}

In this section, we prove that the steady state solutions of (7) and (8) coincide. Furthermore we note that solutions of (7) remain finite and (7) has a unique steady state solution for small force.

The forced Burgers equation

$$
u_{t}+u u_{x}-\nu u_{x x}=F(x)
$$

is transformed to

$$
u_{t}=u_{x x}-u u_{x}+h(x)
$$

by letting $u=\nu \tilde{u}, t=\frac{1}{\nu} \tilde{t}$ and $h(x)=F(x) / \nu^{2}$ so that the viscosity only appears in the forcing term. The mean value of $u$ is given by 


$$
m(t)=\frac{1}{2 \pi} \int_{0}^{2 \pi} u(x, t) d x
$$

and the rate of change of $m$ with respect to time satisfies

$$
\dot{m}(t)=\frac{1}{2 \pi} \int_{0}^{2 \pi} h(x) d x .
$$

The force $h$ will be assumed to have zero mean so that by (11) the mean of $u$ is conserved.

The solution of Equation (7) is treated as a solution of a reaction-diffusion system by introducing a nonlinear change of variables. Let $u$ be a solution of Equation (7) and let $J(u)=\left(u, u_{x},-\frac{1}{2} u^{2}\right)$. Then $(u, v, w)=J(u)$ satisfies Equation (8). The mean of $u$ in (8) is conserved since $h$ has zero mean and the mean of $v$ is also conserved if $h$ satisfies the periodic boundary conditions. However, the mean of $w$ is not conserved. To conserve the mean of $w$, we modify Equation (8) by setting

$$
\widetilde{w}(x, t)=w(x, t)-\frac{1}{2 \pi} \int_{0}^{2 \pi} w(x, t) d x .
$$

The drift-free reaction-diffusion system becomes

$$
\begin{gathered}
u_{t}=u_{x x}+\widetilde{w}_{x}+h(x) \\
v_{t}=v_{x x}+\widetilde{w}_{x x}+h^{\prime}(x) \\
\widetilde{w}_{t}=\widetilde{w}_{x x}+v^{2}+u^{2} v-u h(x)-\frac{1}{2 \pi} \int_{0}^{2 \pi}\left(u_{x}^{2}-u h\right) d x
\end{gathered}
$$

Lemma 3.1: If $v(x, 0)=u_{x}(x, 0)$, then $v(x, t)=u_{x}(x, t) \forall t \geq 0$.

Proof: Let $\eta=v-u_{x}$. Then $\eta_{t}=\eta_{x x}$ with $\eta(x, 0)=0$. The uniqueness property of solutions to the diffusion equation with periodic boundary conditions and zero mean implies that $\eta \equiv 0$; hence $v(x, t)=u_{x}(x, t)$.

Lemma 3.2: For any steady state solutions of (8), $u_{x}=v$.

Proof: Let $\eta(x)=u_{x}-v$. Then $\eta$ satisfies $\eta_{x x}=0$. Since $\eta$ is periodic in space with zero mean, $\eta \equiv 0$.

Lemma 3.3: For any steady state solutions of (8),

$$
\frac{1}{2 \pi} \int_{0}^{2 \pi}\left(u_{x}^{2}-u h\right) d x=0
$$

Proof: From (8),

$$
\int_{0}^{2 \pi} w_{t}(x, t) d x=\int_{0}^{2 \pi}\left(w_{x x}+u^{2} v\right) d x+\int_{0}^{2 \pi}\left(v^{2}-u h\right) d x .
$$


Lemma 3.1 implies that

$$
\int_{0}^{2 \pi} w_{t}(x, t) d x=\left[w_{x}+\frac{1}{3} u^{3}\right]_{0}^{2 \pi}+\int_{0}^{2 \pi}\left(u_{x}^{2}-u h\right) d x
$$

Using the periodicity of $u$ and $v$, the result

follows at the steady state.

$$
\int_{0}^{2 \pi}\left(u_{x}^{2}-u h\right) d x=0
$$

Lemma 3.4: Let

$$
\begin{gathered}
\widetilde{w}(x, t)=w(x, t)-\frac{1}{2 \pi} \int_{0}^{2 \pi} w(x, t) d x, \\
\tilde{\xi}(x, t)=\widetilde{w}(x, t)+\frac{1}{2} u^{2}(x, t)
\end{gathered}
$$

and

$$
\xi(x, t)=w(x, t)+\frac{1}{2} u^{2}(x, t)
$$

Then $\widetilde{\xi}=\xi$ at the steady state.

Proof: Since $\int_{0}^{2 \pi} w(x, t) d x$ is independent of $x$, we have $\widetilde{w}_{x}=w_{x}$ and $\widetilde{w}_{x x}=w_{x x}$. Therefore, using (19), (20) and (21), we get

and

$$
\begin{gathered}
\xi_{x}=u u_{x}+w_{x}=\widetilde{\xi}_{x} \\
\xi_{x x}=\left(u_{x}\right)^{2}+u u_{x x}+w_{x x}=\widetilde{\xi}_{x x}
\end{gathered}
$$

$$
\xi_{t}=u u_{t}+w_{t}=\tilde{\xi}_{t}+\frac{1}{2 \pi} \int_{0}^{2 \pi} w_{t}(x, t) d x .
$$

Using Equation (14) we get

$$
\xi_{t}=u \xi_{x}+\xi_{x x}+\eta u^{2}+v^{2}-u_{x}^{2}
$$

where $\eta=v-u_{x}$. By Lemma 3.1, $\eta=0$. Hence, $v^{2}=u_{x}^{2}$ and $\xi_{t}=u \xi_{x}+\xi_{x x}$. Thus,

$$
\tilde{\xi}_{t}=u \tilde{\xi}_{x}+\tilde{\xi}_{x x}-\frac{1}{2 \pi} \int_{0}^{2 \pi} w_{t}(x, t) d x
$$

By Lemma 3.3,

$$
\tilde{\xi}_{t}=u \tilde{\xi}_{x}+\tilde{\xi}_{x x}-\frac{1}{2 \pi} \int_{0}^{2 \pi}\left(u_{x}^{2}-u h\right) d x
$$

In the steady state, the result 


$$
\tilde{\xi}=\xi
$$

follows from the fact $u \xi_{x}+\xi_{x x}=0=u \tilde{\xi}_{x}+\tilde{\xi}_{x x}$, and $u$ has zero mean.

Now we will prove that the steady state solution of the forced Burgers equation is also the steady state solution of the transformed reaction-diffusion system and conversely.

Theorem 3.1: The steady state solution of the forced Burgers equation

$$
u_{t}=u_{x x}-u u_{x}+h(x)
$$

is also the steady state solution to the transformed reaction-diffusion system of the Burgers equation:

$$
\begin{gathered}
u_{t}=u_{x x}+\widetilde{w}_{x}+h(x) \\
v_{t}=v_{x x}+\widetilde{w}_{x x}+h^{\prime}(x) \\
\widetilde{w}_{t}=\widetilde{w}_{x x}+u^{2} v+v^{2}-u h(x)-\frac{1}{2 \pi} \int_{0}^{2 \pi}\left(u_{x}^{2}-u h\right) d x .
\end{gathered}
$$

Conversely, any steady state solution $(u, v, \widetilde{w})$ of $(30)$ is necessarily of the form $v=$ $u_{x}, \widetilde{w}=w$ with $w=-\frac{1}{2} u^{2}$, and $u$ being a steady state solution of (29).

Proof: Because $v_{t}=\widetilde{w}_{t}=0$, it follows from (30) and (15) that

$$
v_{x x}=u^{2} v+v^{2}-u h(x)-h^{\prime}(x)=0 .
$$

Since $v=u_{x}$, Equation (31) becomes

$$
u_{x x x}-u^{2} u_{x}-u_{x}^{2}+u h(x)+h^{\prime}(x)=0
$$

which implies

$$
\left(u_{x x}-u u_{x}+h(x)\right)_{x}+u\left(u_{x x}-u u_{x}+h(x)\right)=0
$$

However,

$$
u_{x x}-u u_{x}+h(x)=0
$$

since $u$ is a steady state solution of the forced Burgers equation. Similar arguments hold in the case where $h(x)=0$.

To prove the converse, observe that the steady state solution of (30) satisfies

$$
\begin{gathered}
u_{x x}+\widetilde{w}_{x}+h(x)=0 \\
v_{x x}+\widetilde{w}_{x x}+h^{\prime}(x)=0 \\
\widetilde{w}_{x x}+u^{2} v+v^{2}-u h(x)=0 .
\end{gathered}
$$

By subtracting the last two equations in (35), one obtains

$$
v_{x x}+h^{\prime}(x)-u^{2} v-v^{2}+u=0 .
$$


Since $v=u_{x}$, from Lemma 3.1, (36) becomes

$$
u_{x x x}+h^{\prime}(x)-u^{2} u_{x}-u_{x}^{2}+u h(x)=0
$$

which can be written as

$$
\left(u_{x x}-u u_{x}+h(x)\right)_{x}+u\left(u_{x x}-u u_{x}+h(x)\right)=0
$$

Let

Then

$$
\phi=u_{x x}-u u_{x}+h(x)
$$

$$
\phi_{x}+u \phi=0
$$

If

$$
\theta=\exp \left(\int_{0}^{x} u(s) d s\right)
$$

then

$$
(\phi \theta)_{x}=0
$$

which implies that $\phi=c_{1} / \theta$.

$$
\begin{gathered}
\int_{0}^{2 \pi} \phi(x) d x=\int_{0}^{2 \pi}\left(u_{x x}-u u_{x}+h(x)\right) d x \\
=\int_{0}^{2 \pi}\left(u_{x}-\frac{u^{2}}{2}\right)_{x} d x+\int_{0}^{2 \pi} h(s) d s
\end{gathered}
$$

Using the periodicity of $u$ and the fact that $\int_{0}^{2 \pi} h(s) d s=0$, we get

$$
\int_{0}^{2 \pi} \phi(s) d s=0
$$

which implies $\int_{0}^{2 \pi^{c}} \frac{1}{\theta} d x=0$. Since $\theta>0$, we have $c_{1}=\phi=0$ and

$$
u_{x x}-u u_{x}+h(x)=0
$$

Theorem 3.2: Every solution to the forced Burgers equation

$$
u_{t}=u_{x x}-u u_{x}+h(x)
$$

satisfies the inequality

$$
\|u\|_{L_{(0,2 \pi)}^{2}} \leq \sqrt{2} c\|h\|_{L_{(0,2 \pi)}^{2}}, \text { for } t \geq t_{0}, \text { with } t_{0}=c \ln \left\{\frac{\int_{0}^{2 \pi} u_{0}^{2} d x}{c^{2} \int_{0}^{2 \pi} h^{2} d x}\right\}
$$

and $c$ being the Poincare constant. 
Proof: If we multiply the above equation by $u$ and integrate, we get

$$
\frac{d}{d t}\left(\frac{1}{2} \int_{0}^{2 \pi} u^{2} d x\right)=\int_{0}^{2 \pi} u u_{x x} d x+\int_{0}^{2 \pi} u h d x
$$

Since $u$ is periodic, Equation (48) becomes

$$
\frac{d}{d t}\left(\frac{1}{2} \int_{0}^{2 \pi} u^{2} d x\right)=-\int_{0}^{2 \pi} u_{x}^{2} d x+\int_{0}^{2 \pi} u h d x .
$$

Then using the Poincare inequality on (49) and the zero mean condition on $u$, we get

$$
\frac{d}{d t}\left(\frac{1}{2} \int_{0}^{2 \pi} u^{2} d x\right) \leq-\frac{1}{c} \int_{0}^{2 \pi} u^{2} d x+\int_{0}^{2 \pi} \frac{1}{\sqrt{c}} u \sqrt{c} h d x
$$

and the Cauchy Schwartz inequality on (50) to obtain

$$
\frac{1}{2} \frac{d}{d t}\left(\int_{0}^{2 \pi} u^{2} d x\right)+\frac{1}{c} \int_{0}^{2 \pi} u^{2} d x \leq\left(\int_{0}^{2 \pi} \frac{u^{2}}{c} d x\right)^{1 / 2}\left(\int_{0}^{2 \pi} c h^{2} d x\right)^{1 / 2}
$$

Also, using the inequality $a b<\frac{a^{2}}{2}+\frac{b^{2}}{2}$, Equation (51) becomes

$$
\frac{d}{d t}\left(\int_{0}^{2 \pi} u^{2} d x\right)+\frac{1}{c} \int_{0}^{2 \pi} u^{2} d x \leq c \int_{0}^{2 \pi} h^{2} d x
$$

Finally, using the Gronwall inequality on (52) we arrive at

$$
\int_{0}^{2 \pi} u^{2} d x \leq e^{-\frac{t}{c}} \int_{0}^{2 \pi} u_{0}^{2} d x+c^{2}\left(1-e^{-\frac{t}{c}}\right) \int_{0}^{2 \pi} h^{2} d x
$$

Given $\int_{0}^{2 \pi} u_{0}^{2} d x$, for $t \geq t_{0}$ with $t_{0}=c \ln \left\{\frac{\int_{0}^{2 \pi} u_{0}^{2} d x}{c^{2} \int_{0}^{2 \pi} h^{2} d x}\right\}$, we have that

$$
\int_{0}^{2 \pi} u^{2} d x \leq 2 c^{2} \int_{0}^{2 \pi} h^{2} d x
$$

which implies that

$$
\|u\|_{L_{(0,2 \pi)}^{2}} \leq \sqrt{2} c\|h\|_{L_{(0,2 \pi)}^{2}}
$$

Inequality (55) can be refined for the steady state, since from (52) it follows that 


$$
\|u\|_{L_{(0,2 \pi)}^{2}} \leq c\|h\|_{L_{(0,2 \pi)}^{2}}
$$

Hence, we have the first part of Lemma 3.5 proved.

Lemma 3.5: The steady state solution $u$ of the forced Burgers equation satisfies the following inequalities:

$$
\begin{gathered}
\|u\|_{L_{(0,2 \pi)}^{2}} \leq c\|h\|_{L_{(0,2 \pi)}^{2}} \\
\left\|u_{x}\right\|_{L_{(0,2 \pi)}^{2}} \leq \sqrt{c}\|h\|_{L_{(0,2 \pi)}^{2}}
\end{gathered}
$$

Proof: Since

$$
u_{x x}-u u_{x}+h=0
$$

we can multiply Equation (59) by $u$, integrate the result from 0 to $2 \pi$, and use the periodicity of $u$ to obtain

$$
\left\|u_{x}\right\|_{L_{(0,2 \pi)}^{2}}^{2} \leq\|u\|_{L_{(0,2 \pi)}^{2}} \cdot\|h\|_{L_{(0,2 \pi)}^{2}} .
$$

Since $\|u\|_{L_{(0,2 \pi)}^{2}} \leq c\|h\|_{L_{(0,2 \pi)}^{2}}$, Equation (60) becomes

$$
\left\|u_{x}\right\|_{L_{(0,2 \pi)}^{2}} \leq \sqrt{c}\|h\|_{L_{(0,2 \pi)}^{2}} .
$$

Theorem 3.3: $\quad$ There is a unique steady state solution to the forced Burgers equation

$$
u_{t}=u_{x x}-u u_{x}+h(x)
$$
when $h$ satisfies $\|h\|_{L_{(0,2 \pi)}^{2}}<2 /\left(3 c_{1} c\right), c$ is the Poincare constant, and $c_{1}$ is the
Sobolev constant.

Proof: Suppose there are two solutions $u$ and $v$ such that

and

$$
u_{x x}-u u_{x}+h(x)=0
$$

Let $w=u-v$. Then,

$$
v_{x x}-v v_{x}+h(x)=0
$$

$$
w_{x x}-u w_{x}-w v_{x}=0
$$

Multiplying the above equation by $w$, integrating from 0 to $2 \pi$, and using the periodicity of $u$ and $w$ leads to

$$
\int_{0}^{2 \pi}\left(w_{x}\right)^{2} d x=\int_{0}^{2 \pi} w^{2}\left(\frac{u_{x}}{2}-v_{x}\right) d x .
$$

The latter can be rewritten as 


$$
\left\|w_{x}\right\|_{L_{(0,2 \pi)}^{2}}^{2} \leq\left\|w^{2}\right\|_{L_{(0,2 \pi)}^{2}}\left(\frac{1}{2}\left\|u_{x}\right\|_{L_{(0,2 \pi)}^{2}}+\left\|v_{x}\right\|_{L_{(0,2 \pi)}^{2}}\right)
$$

and by (58) in the form

Since

$$
\left\|w_{x}\right\|_{L_{(0,2 \pi)}^{2}}^{2} \leq \frac{3 \sqrt{c}}{2}\|h\|_{L_{(0,2 \pi)}^{2}} \cdot\left\|w^{2}\right\|_{L_{(0,2 \pi)}^{2}} .
$$

it follows that

$$
\left\|w^{2}\right\|_{L_{(0,2 \pi)}^{2}} \leq c_{1}\|w\|_{L_{(0,2 \pi)}^{\infty}}^{2} \leq c_{1}\|w\|_{L_{(0,2 \pi)}^{2}} \cdot\left\|w_{x}\right\|_{L_{(0,2 \pi)}^{2}},
$$

$$
\left\|w_{x}\right\|_{L_{(0,2 \pi)}^{2}} \leq\left(\frac{3 c c_{1}}{2}\right)^{1 / 2}\|h\|_{L_{(0,2 \pi)}^{2}}^{\frac{1}{2}} \cdot\left\|w_{x}\right\|_{L_{(0,2 \pi)}^{2}}
$$

If $\|h\|_{L_{(0,2 \pi)}^{2}}<\left(\frac{3}{2} c c_{1}\right)^{-1}$ then $w=w_{x}=0$, which implies $u=v$.

\section{Numerical Results}

\subsection{Fourier Representation of the Transformed Burgers Equations}

The quasilinear parabolic equation

$$
u_{t}=u_{x x}-u u_{x}+h(x)
$$

with $u(2 \pi, t)=u(0, t)$ and $u(x, 0)=u_{0}(x)$ can be written as

$$
\frac{\partial u}{\partial t}=G(u, h)
$$

where $G(u, h)=u_{x x}-u u_{x}+h(x)$. The discretization process consists of defining a space $X_{N}$ of trial functions, a space $Y_{N}$ of test functions, discrete approximations $G_{N}$ of the operator $G$, and an orthogonal projection operator $Q_{N}$ from a suitable Hilbert space, which contains $X_{N}$ onto the space $Y_{N}$. We choose the spaces $X_{N}$ and $Y_{N}$ to be the space $S_{N}$ of all trigonometric polynomials of degree $\leq N / 2$. If $u^{N} \in$ $S_{N}$, then

$$
u^{N}(x, t)=\sum_{k=-N / 2}^{N / 2-1} \widehat{u}_{k}(t) e^{i k x}
$$

where $\widehat{u}_{k}(t), k=-N / 2, \ldots, N / 2-1$ are the Fourier coefficients. If the residual of (71) is orthogonal to all test functions in $S_{N}$, then a set of ODEs will be obtained. The scalar Burgers equation in the Fourier space can be written as

$$
\widehat{u}_{t}(t, k)=-k^{2} \widehat{u}(t, k)-\sum_{p+q=k} \widehat{u}(t, p) \widehat{u}(t, q)+\frac{1}{\nu^{2}} \widehat{F}(k)-\sum_{p+q=k \pm N} \widehat{u}(t, p) \widehat{u}(t, q) .
$$


The transformed Burgers equation is

$$
\begin{gathered}
u_{t}=u_{x x}+w_{x}+h(x) \\
v_{t}=v_{x x}+w_{x x}+h^{\prime}(x) \\
w_{t}=w_{x x}+u^{2} v+v^{2}-u h(x),
\end{gathered}
$$

where $v(x, t)=u_{x}(x, t), w(x, t)=-\frac{1}{2} u^{2}(x, t)$ and $h(x)=F(x) / \nu^{2}$, with $u(2 \pi, t)=$ $u(0, t)$ and $u(x, 0)=u_{0}(x)$. The rate of change with respect to the time of the mean of $u$ and $v$ in the above system is zero, but that of $w$ is different from zero. We modify (75) so that the drift in the mean of $w$ is normalized to 0 , i.e.,

$$
\widetilde{w}(x, t)=w(x, t)-\frac{1}{2 \pi} \int_{0}^{2 \pi} w(x, t) d x
$$

The drift-free transformed Burgers equation is now

$$
\begin{gathered}
u_{t}=u_{x x}+\widetilde{w}_{x}+h(x) \\
v_{t}=v_{x x}+\widetilde{w}_{x x}+h^{\prime}(x) \\
\widetilde{w}_{t}=\widetilde{w}_{x x}+u^{2} v+v^{2}-u h(x)-\frac{1}{2 \pi} \int_{0}^{2 \pi}\left(u_{x}^{2}-u h\right) d x .
\end{gathered}
$$

If $u^{N} \in S_{N}, v^{N} \in S_{N}$, and $\widetilde{w}^{N} \in S_{N}$, then

$$
\begin{gathered}
u^{N}(x, t)=\sum_{k=-N / 2}^{N / 2-1} \widehat{u}_{k}(t) e^{i k x} \\
v^{N}(x, t)=\sum_{k=-N / 2}^{N / 2-1} \widehat{v}_{k}(t) e^{i k x}
\end{gathered}
$$

and

$$
\widetilde{w}^{N}(x, t) \sum_{k=-N / 2}^{N / 2-1} \hat{\widetilde{w}}_{k}(t) e^{i k x}
$$

The transformed system in the Fourier space is

$$
\begin{gathered}
\widehat{u}_{t}(t, k)=-k^{2} \widehat{u}(t, k)+i k \widehat{\widetilde{w}}(t, k)+\widehat{h}(k) \\
\widehat{v}_{t}(t, k)=-k^{2} \widehat{v}(t, k)-k^{2} \widehat{\widetilde{w}}(t, k)+i k \hat{h}(k) \\
\widehat{\widetilde{w}}_{t}(t, k)=-k^{2} \widehat{\widetilde{w}}(t, k)+\sum_{p+q=k} \widehat{u}(t, p) \cdot \widehat{u}(t, q)+\sum_{p+q+l=k} \widehat{u}(t, p) \widehat{u}(t, l) \widehat{v}(t, q)
\end{gathered}
$$




$$
\begin{gathered}
-\sum_{p+q=k} \hat{h}(q) \widehat{u}(t, p)-\sum_{p+q=k \pm N} \hat{h}(q) \widehat{u}(t, p) \\
+\sum_{p+q=k \pm N} \hat{u}(t, p) \widehat{u}(t, q)+\sum_{p+q+l=k \pm N} \widehat{u}(t, l) \widehat{u}(t, p) \widehat{v}(t, q)-\hat{m}(t),
\end{gathered}
$$

where $m(t)=\frac{1}{2 \pi} \int_{0}^{2 \pi}\left(u_{x}^{2}-u h\right) d x$. In the transformation method, all the nonlinear terms in (74) and (81) were evaluated by performing all the multiplications in a physical space followed by the discrete Fourier transform to determine the corresponding Fourier coefficients. The aliasing error was removed by truncation, as it will be described in the next section.

\subsection{Aliasing Removal by Truncation}

The aliasing removal by truncation in the scalar Burgers equation proceeds in the manner described in [6], which is the " $2 / 3$ rule". In the transformed system, the $2 / 3$ rule is not appropriate because of the third order nonlinearity in the third equation of the system. The "de-aliasing" technique that is used in the transformed system involves the use of the discrete transform with $M$ rather than $N$ points, where $M \geq 2 N$. Let

$$
\begin{gathered}
x_{j}=2 \pi j / M, j=0,1, \ldots, M-1 \\
\bar{U}_{j}=\sum_{k=-M / 2}^{M / 2-1} \tilde{u}_{k} e^{i k x} j \\
\bar{V}_{j}=\sum_{k=-M / 2}^{M / 2-1} \tilde{v}_{k} e^{i k x_{j}} \\
\bar{W}_{j}=\sum_{k=-M / 2}^{M / 2-1} \tilde{w}_{k} e^{i k x} j \\
U_{j}=\bar{U}_{j} \bar{V}_{j} \bar{W}_{j},
\end{gathered}
$$

where

$$
\begin{aligned}
& \tilde{u}_{k}=\left\{\begin{array}{cc}
\hat{u}_{k} & \text { if }|k| \leq N / 2 \\
0 & \text { otherwise }
\end{array}\right. \\
& \tilde{v}_{k}=\left\{\begin{array}{cc}
\hat{v}_{k} & \text { if }|k| \leq N / 2 \\
0 & \text { otherwise }
\end{array}\right.
\end{aligned}
$$

and 


$$
\widetilde{w}_{k}=\left\{\begin{array}{cc}
\widehat{w}_{k} & \text { if }|k| \leq N / 2 \\
0 & \text { otherwise. }
\end{array}\right.
$$

Thus, the coefficients $\tilde{u}_{k}, \widetilde{v}_{k}$ and $\widetilde{w}_{k}$ are the coefficients $\widehat{u}_{k}, \widehat{v}_{k}$, and $\widehat{w}_{k}$ padded with zeros for the additional wavenumbers. Similarly, let

$$
\widetilde{U}_{k}=\frac{1}{M} \sum_{j=0}^{M-1} U_{j} e^{-i k x} j ; \quad k=-M / 2, \ldots, M / 2-1 .
$$

Then

$$
\widetilde{U}_{k}=\sum_{m+l+p=k} \tilde{u}_{m} \widetilde{v}_{l} \widetilde{w}_{p}+\sum_{m+l+p=k \pm M} \tilde{u}_{m} \widetilde{v}_{l} \widetilde{w}_{p}
$$

Since we are only interested in $\tilde{U}_{k}$ for $|k| \leq N / 2$, we can choose $M$ such that the second term on the right-hand side vanishes for these values of $k$. Since $\tilde{u}_{m}, \widetilde{v}_{m}$ and $\widetilde{w}_{m}$ are zero for $|m|>N / 2$, the worst case condition is

$$
M \geq 2 N-1
$$

If $M$ is chosen as above, then the aliasing error in all terms of the third order and less will be zero. This is the "2-rule" de-aliasing technique used in the reaction-diffusion system.

Two computer programs have been written to solve (74) and (81). In the first, a spectral Galerkin method with $N=256$ is used. The Fourier coefficients, for which $|k| \geq(1 / 3) N$, are set to zero at each time step so that the aliasing term in (74) vanishes. In the second program, a spectral Galerkin method with $N=256$ is also used. The Fourier coefficients, for which $|k| \geq(1 / 4) N$ are set to zero at each time step so that the aliasing term in (81) vanishes.

The integration is done using the spectral Galerkin method described above with the "slaved-frog" as a temporal scheme [11], i.e.,

$$
q_{n+1}=e^{-2 \alpha \delta t} q_{n-1}+\left(\frac{1-e^{-2 \alpha \delta t}}{\alpha}\right) f_{n}
$$

where $q_{n}=q\left(t_{n}\right), f_{n}=f\left(t_{n}\right)$. This is obtained from the exact relation

$$
q(t+\delta t)=e^{-2 \alpha \delta t} q(t-\delta t)+\int_{t-\delta t}^{t+\delta t} e^{-\alpha(t+\delta t-s)} f(s) d s
$$

This scheme reduces to the "leapfrog" scheme when $\alpha=0$. It is a second order in time and unconditionally stable when $f=0$.

Figure 1 depicts the time evolution of $u$ for the forced scalar Burgers equation with $F(x)=3 \cos x$ and $u(x, 0)=\sin x$ and Figure 2 describes the time evolution of $u$ for the transformed system. 


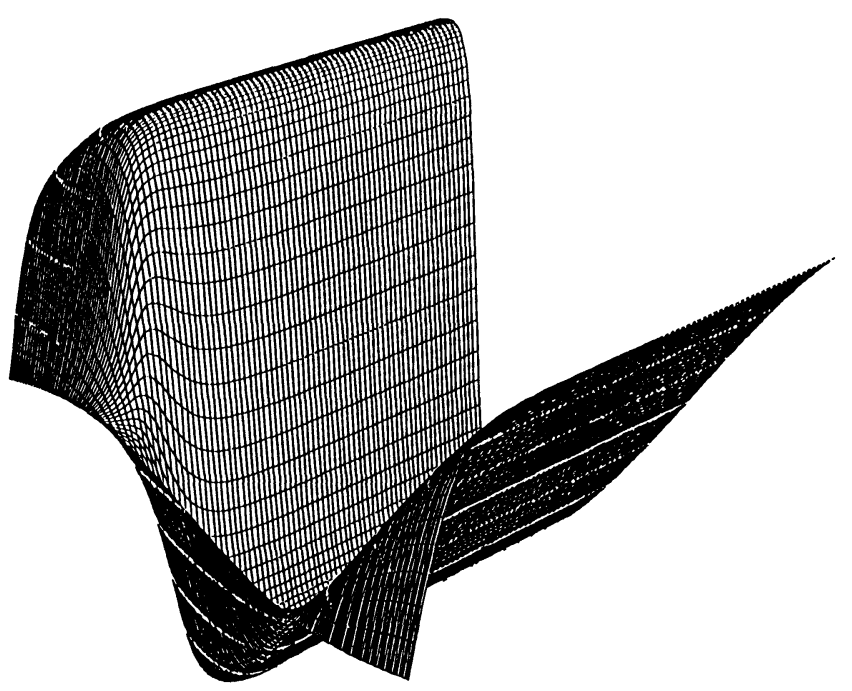

Figure 1. The time evolution of $u$ for the scalar Burgers equation (86) with $F(x)=3 \cos x, \delta t=0.0002$ and $u(x, 0)=\sin x$. Output is every 200 time steps.

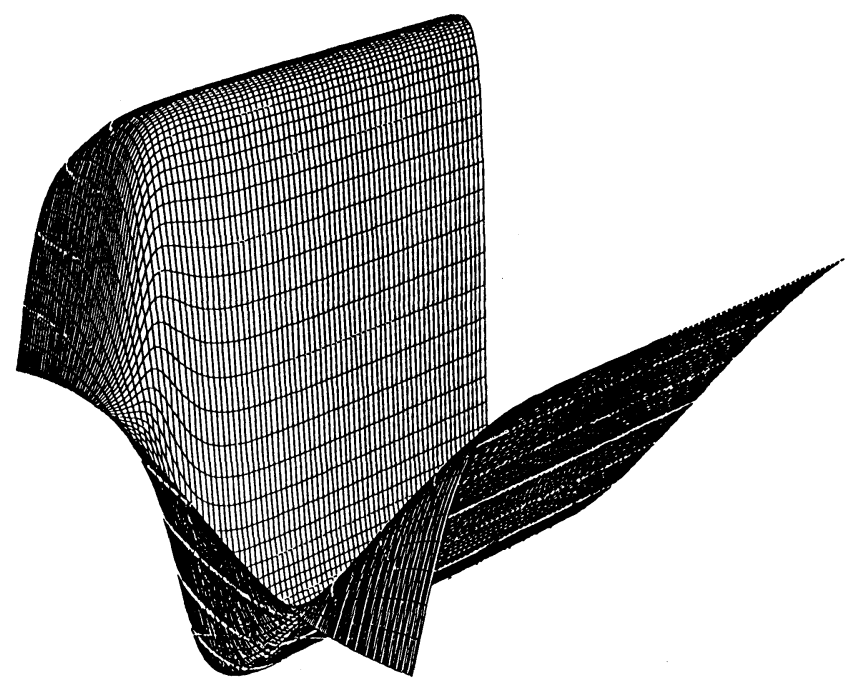

Figure 2. The time evolution of $u$ for the transformed system (92) with $F(x)=3 \cos x, \delta t=0.0002$ and $u(x, 0)=\sin x$. Output is every 200 time steps.

In both cases, the steady state solutions converge with at least four accurate digits in $10^{4}$ time steps, depending on $\nu$ (when $\nu$ is large, there is a critical slow down). The steady state solutions of the scalar Burgers equation was also used as the initial condition for the system and vice versa. After only one time step, the four digits of accuracy were observed. Figures 3 and 4 show the time evolution of $u$ for the scalar Burgers equation and for the transformed system, respectively, but with a different 
forcing $F(x)=3 \cos 2 x$.

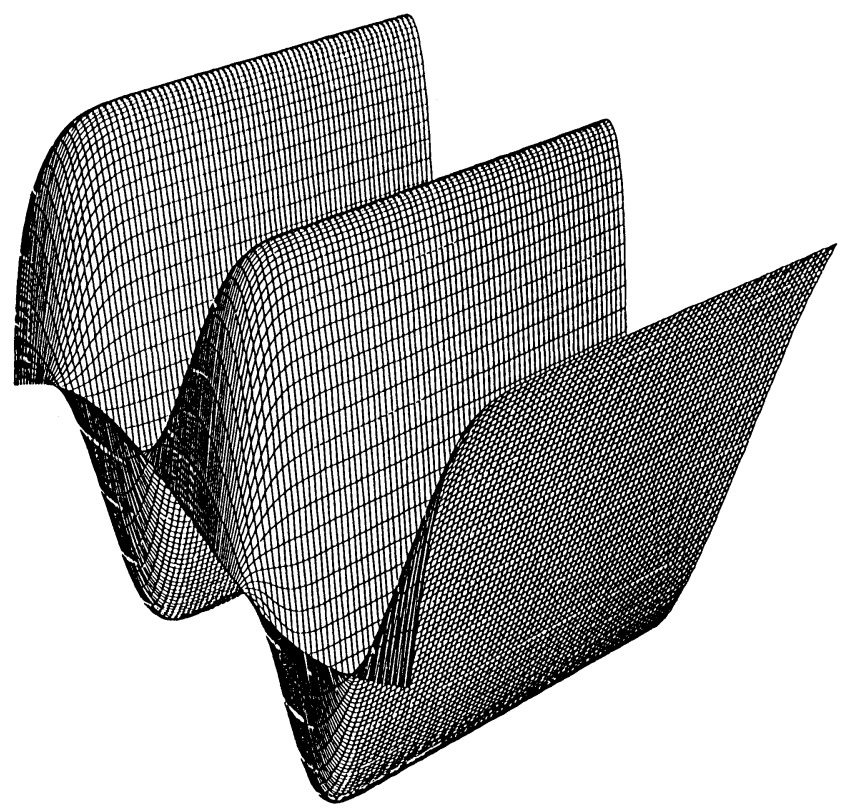

Figure 3. The time evolution of $u$ for the scalar Burgers equation (86) with $F(x)=3 \cos 2 x, \delta t=0.0002$ and $u(x, 0)=\sin x$. Output is every 200 time steps.

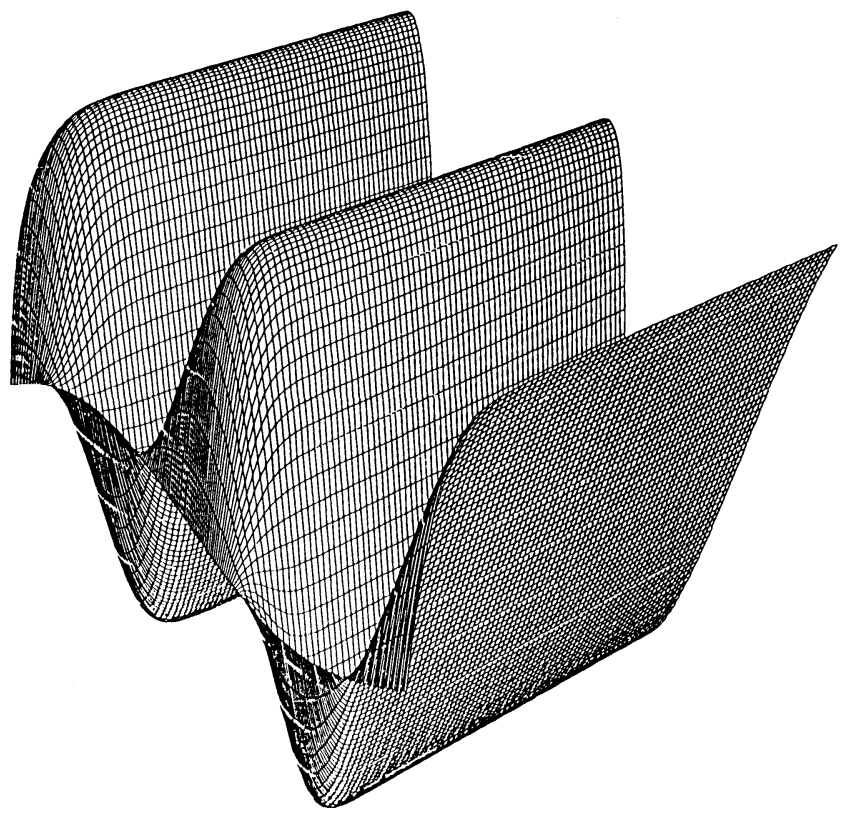

Figure 4. The time evolution of $u$ for the transformed system (92) with $F(x)=3 \cos 2 x, \delta t=0.0002$ and $u(x, 0)=\sin x$. Output is every 200 time steps. 
Other sinusoidal forcing terms were used and similar results were obtained. Thus, one can conclude that both analytical and numerical results presented here show that if Burgers equation is transformed to a reaction diffusion system, then the two systems have similar long time dynamical behavior. Hence this work not only supports Kwak's theory on the existence of inertial manifold for the 2-D NavierStokes equation, but also opens up a new numerical approach to study the dynamics of more complicated PDE's.

\section{Acknowledgments}

The author would like to acknowledge helpful conversations with Professors Alp Eden and Basil Nicolaenko. Also, he would like to thank the anonymous referee for his/her valuable comments, which improved the paper significantly.

\section{References}

[1] Babin, A.V. and Vishik, M.I., Attractors of partial differential equations and estimate of their dimension, Uspeki Mat. Nauk. 38 (1983), 133-187. (In Russia) Russian Math. Surveys 28 (1983), 151-213. (In English)

[2] Bateman, H., Some recent researches on the motion of fluids, Mon. Weather Rev. 43 (1915), 163-170.

[3] Blackstock, D.T., Convergence of the Keck-Boyer perturbation solution for plane waves of finite amplitude in a viscous fluid, J. Acoust. Soc. Am. 39 (1966), 411-413.

[4] Burgers, J.M., A mathematical model illustrating the theory of turbulence, Adv. Appl. Mech. 1 (1948), 171-199.

[5] Burgers, J.M., The Nonlinear Diffusion Equation, Reidel, Boston 1974.

[6] Canuto, C., Hussaini, M.Y., Quarteroni, A., and Zang, T.A., Spectral Methods in Fluid Dynamics, Springer Series in Computational Physics 1988.

[7] Constantin, C., Foias, C. and Temam, R., On the dimension of the attractors in two-dimensional turbulence, Physica D 30:3 (1988), 284-296.

[8] Foias, C., Nicolaenko, B., Sell, G. and Temam, R., Inertial manifolds for the Kuramoto-Sivashinsky equation and an estimate of their lowest dimension, IMA Preprint Series, J. Math. Pures Appl. 279 (1988).

[9] Foias, C., Sell, G. and Temam, R., Inertial manifolds for nonlinear evolutionary equations, IMA Preprint Series, J. Differential Equations 234 (1986).

[10] Foias, C. and Temam, R., On the Hausdorff dimension of an attractor for the two-dimensional Navier Stokes equations, Phys. Lett. 93:9 (1983), 451-454.

[11] Frisch, U., Su, S.Z. and Thual, O., Viscoelastic behavior of cellular solutions to the Kuramoto-Sivashinsky model, J. Fluid Mech. 168 (1986), 221-240.

[12] Henry, D., Geometric Theory of Semilinear Parabolic Equations, Lect. Notes in Math. 840, Springer-Verlag, New York 1981.

[13] Hopf, E., The partial differential equation $u_{t}+u u_{x}=\nu u_{x x}$, Comm. Pure Appl. Math. 3 (1950), 201-230.

[14] Kwak, M., Finite dimensional inertial form of the two-dimensional Navier Stokes equations, Ind. Math. J. 41 (1992), 927-981.

[15] Ladyzhenskaya, O.A., On the dimension of bounded invariant sets for the 
Navier Stokes equations and other related dissipative systems, J. Soviet. Math. 28:5 (1982), 714-726. (English transl.)

[16] Lighthill, M.J., Viscosity effects in sound waves of finite amplitude, In: Surveys in Mechanics (ed by G.K. Batchelor and R. Davis), Cambridge University Press, Cambridge 1956.

[17] Mallet-Paret, J., Negatively invariant sets of compact maps and an extension of a theorem of Cartwright, J. Differ. Eqns. 22 (1976), 331.

[18] Mañé, R., On the Dimension of the Compact Invariant Sets of Certain Nonlinear Maps, Lecture Notes in Math. 898, Springer-Verlag, New York 1981.

[19] Smaoui, N. and Armbruster, D., Symmetry and the Karhunen-Loeve analysis, SIAM J. Sci. Comput. 18:5 (1997), 1526-1532.

[20] Temam, R., Navier Stokes Equation and Nonlinear Functional Analysis, CBMSNSF Regional Conference Series in Applied Mathematics, SIAM, Philadelphia 1983. 


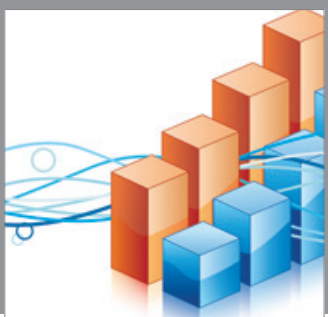

Advances in

Operations Research

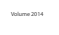

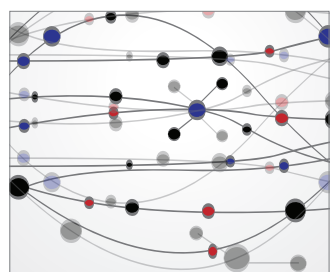

\section{The Scientific} World Journal
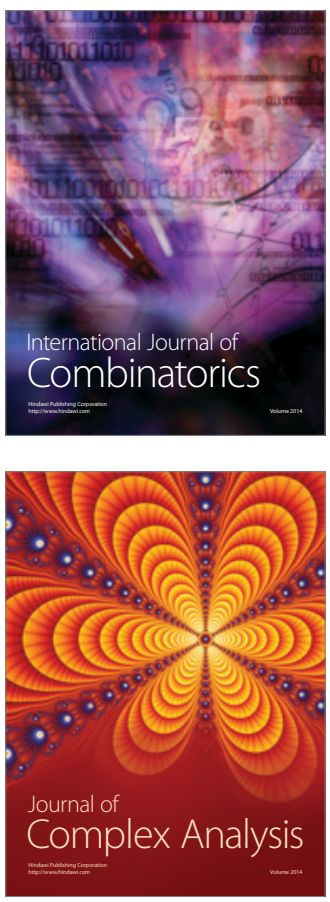

International Journal of

Mathematics and

Mathematical

Sciences
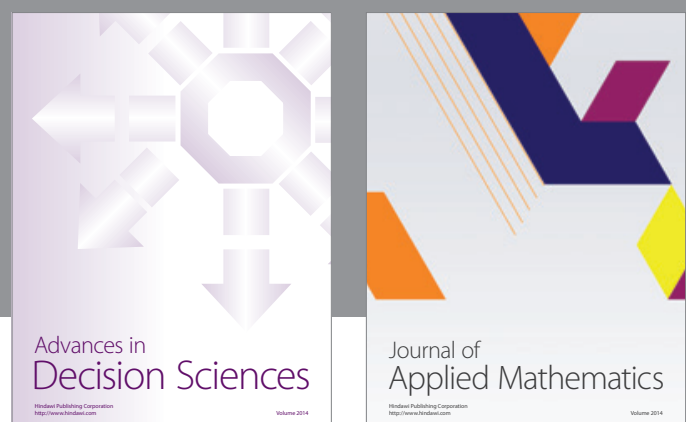

Journal of

Applied Mathematics
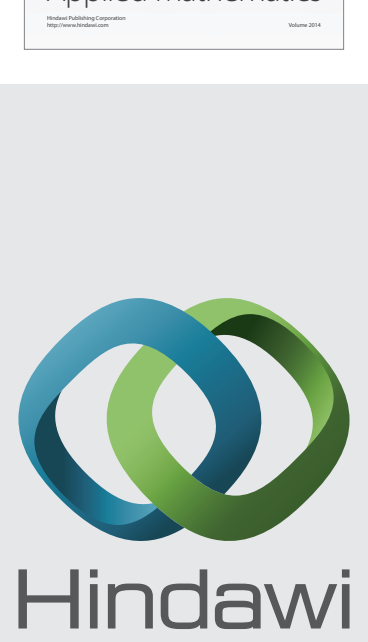

Submit your manuscripts at http://www.hindawi.com
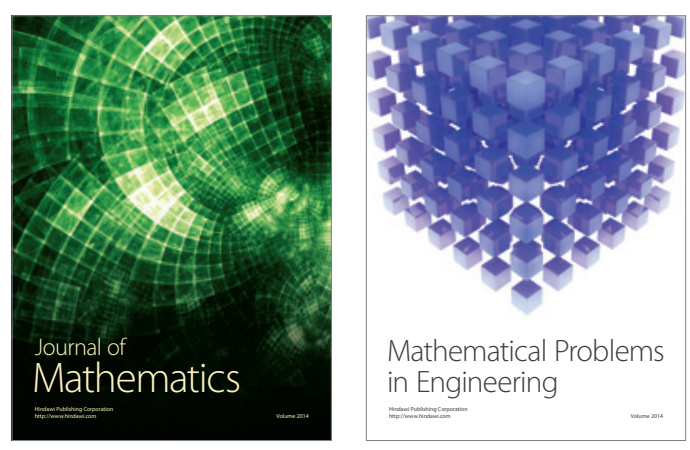

Mathematical Problems in Engineering
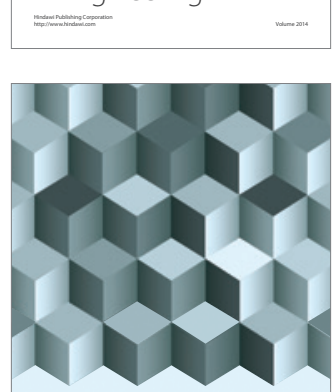

Journal of

Function Spaces
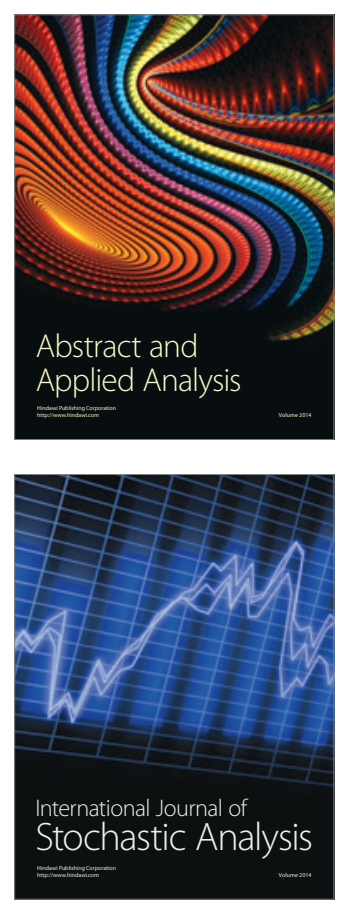

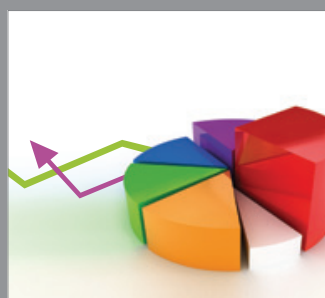

ournal of

Probability and Statistics

Promensencen
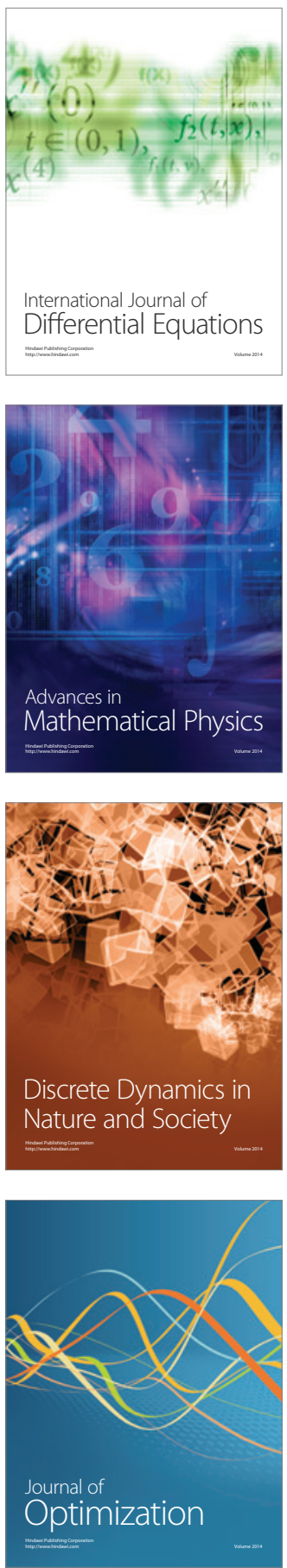